\title{
Clinical and Trichoscopic Study of Tinea Capitis versus Alopecia Areata in Pediatric Patients
}

\author{
LAILA M. MOHAMMED, M.D.; ABEER A. HODEIB, M.D.; WESAM S. IBRAHIM, M.D. and \\ AYA M. HAGGAG, M.Sc.
}

The Department of Dermatology and Venereology, Faculty of Medicine, Tanta University, Tanta, Egypt

\begin{abstract}
Background: Trichoscopy (hair and scalp dermatoscopy) facilitates the diagnosis of hair and scalp disorders. The aim of this study was to identify the trichoscopic features in diagnosis of Tinea Capitis (TC) and to compare these findings with those of Alopecia Areata (AA).

Material and Methods: The current study included 30 patients with TC and 30 patients with AA. They were recruited from Outpatient Clinics of Dermatology and Venereology Department, Tanta University Hospitals. Clinical examination, laboratory investigations (direct microscopic examination with $10 \%$ potassium hydroxide and fungal culture) were done to confirm clinical diagnosis and dermoscopically aiming at finding the different dermoscopic features of TC and AA.

Results: Short broken hairs, black dots, comma shaped hairs, corkscrew hairs and zigzag shaped hairs were observed in patients with TC. Short vellus hairs, black dots, exclamation marks, white hairs and pig tail re-growing hairs were observed in patients with $\mathrm{AA}$.
\end{abstract}

Conclusion: Comma shaped hairs, zigzag shaped hairs and cork screw were observed only in patients with TC. Yellow dots, exclamation mark hairs and short vellus hairs were observed only in patients with AA. Dermoscopy is rapid and reliable confirmatory test used to differentiate between TC and AA by detecting their characteristic dermoscopic features.

Key Words: Tinea capitis $(T C)-$ Alopecia areata $(A A)-$ Paediatric.

\section{Introduction}

TINEA Capitis (TC) is a fungal infection of the scalp, hair follicles and hair shafts. It is especially common in the pediatric population and under tropical conditions [1]. The highest incidence is seen in children 3-7 years of age [2]. Presence of hyperkeratosis of scalp, seborrhea-like symptoms, excoriation secondary to pruritus, alopecia, broken

Correspondence to: Dr. Laila M. Mohammed, The Department of Dermatology and Venereology, Faculty of Medicine, Tanta University, Tanta, Egypt hair or "black dot" appearance, cervical lymphadenopathy or pustules in a child should alert the dermatologist toward the possibility of TC [3]. The prevalence of various causative fungi varies according to the geographical area being studied. TC can present as non-inflammatory or inflammatory morphological variants [4]. An early diagnosis is important to prevent transmission between children, especially siblings, and also to avoid possible scarring and permanent hair loss. Direct microscopic examination with potassium hydroxide $(\mathrm{KOH})$ and fungal culture for appropriate cases are standards for diagnosis of TC [5]. Trichoscopy may help clinical diagnosis of TC [6].

Alopecia Areata (AA) is a medical condition in which hair is lost from some or all areas of the body, usually from the scalp. Typical first symptom of AA is small bald patches. The underlying skin looks superficially normal. These patches can take many shapes but are most usually round or oval [7]. Trichoscopy have been used as a clinical indicator and for follow-up response of treatment in patients with AA $[8,9]$.

Dermatoscopy is a fast, non-invasive and inexpensive diagnostic tool for recognition of morphological structure that cannot be seen with naked eye. It is used widely in the last three decades, especially on diagnosis of pigmented skin lesions [10]. However, it has been increasingly used in many skin diseases such as psoriasis, hair and nail diseases, parasitic diseases and connective tissue diseases [11,12]. Trichoscopy (hair and scalp dermoscopy) facilitates the diagnosis of hair and scalp disorders [13-16], including AA, tricotillomania, female pattern hair loss, TC and inherited hair shaft dystrophies $[\mathbf{6 , 1 7 , 1 8}]$ 


\section{Patients and Methods}

The study included thirty patients with tinea capitis and thirty patients with alopecia areata who were selected from the Outpatient Clinic of Dermatology and Venereology Department, Tanta University Hospital (from August 2014 to August 2015).

\section{Inclusion criteria:}

- Patients less than 12 years old.

- Patients whose parents accepted to be enrolled in the study and one of their parents signed an informed consent.

\section{Exclusion criteria:}

- Patients receiving any topical or systemic medications.

- Other dermatological diseases in the scalp.

\section{Each patient was subjected to:}

\section{A- Full history taking:}

- Personal history: Including name, age, gender and residence.

- Complaint Lesion of patchy hair loss.

- Present history.

Regarding the analysis of the complaint: Onset, duration and course, location, number, associated symptoms: Itching, tenderness and scaling.

B- Clinical examination and digital photography of any lesion of patchy hair loss:

- Clinical examination of the lesion(s) of patchy hair loss: Site, number: Solitary or multiple, size, morphology, and surface description: Scaling, fissuring and crustations.

- Digital photography of the lesions of patchy hair loss; the camera used in the study is sony: Cyber-shot DSC-WX 300 (20x optical zoom18.2 megapixels).

C- Dermoscopic examination and digital photography of the lesions of patchy hair loss; the dermoscope used: Dermlite II PRO HR (3 Gen, USA): DermLite II PRO HR is the most powerful and brightest Dermlite II model. It can be attached to a camera right out of the box Fig. (1).

D- Microscopic examination and fungal culture of skin scraping and plucked hairs.

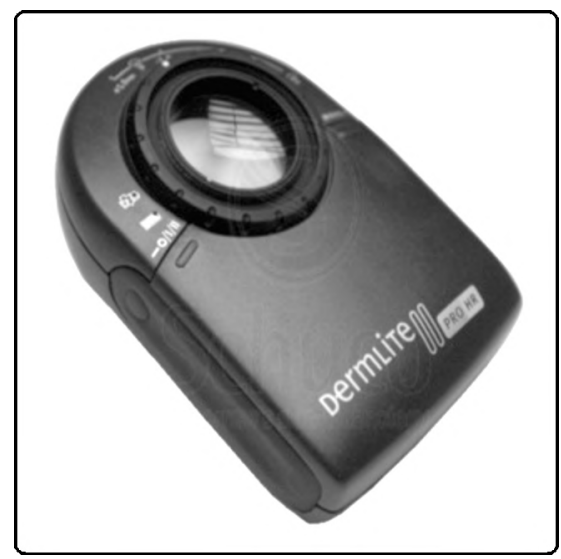

Fig. (1): Dermlite II PRO HR (3Gen, USA) dermoscope.

Specimen collection:

The scalp should be cleaned with alcohol $70 \%$ prior to collection in order to ovoid bacterial contamination. The area of patchy hair loss can be scraped with a blunt surgical blade in order to obtain scales. A forceps can be used to pluck affected hair stubs and black dots hair.

\section{Microscopic examination:}

Hair roots and skin scraping were mounted in $10 \% \mathrm{KOH}$ solution. The slide was gently heated and microscopically examined for spores and hyphae.

\section{Fungal culture:}

The culture was done on Saboraud dextrose agar medium with chloramphenicol and dermasal selective supplement.

The constituents of this medium were as follows: Dextrose 20gm, Peptone 10gm, Agar 20gm, Distilled water 1 litre, 250mg chloramphenicol was added to the media to inhibit growth of bacteria. Dermasal selective supplement (200mg cyclohexamide-25mg chloramphenicol) was added to reduce the growth of saprophytic fungi and to facilitate isolation of dermatophyte. The plates were incubated at $27^{\circ} \mathrm{C}$ for up to four weeks.

Growth on Saboraud dextrose agar was examined for growth, morphology, colony, appearance, surface texture, shape, size, color, rate of growth, edge and the under surface.

Microscopic examination of the suspected colonies was performed to distinguish the fungal elements and identify the type and arrangement of conidia.

\section{Results}

Sixty patients were included in this study. They were collected from the Outpatient Clinics of 
Dermatology and Venereology Department, Tanta University Hospital. They were subdivided into 2 groups; 30 patients had alopecia and 30 patients had TC.

\section{I- Clinical results:}

Out of 30 patients with alopecia, 26 patients $(86.67 \%)$ had AA, 3 patients (10\%) had ophiasis and only one patient (3.33\%) had AU Fig. (2).

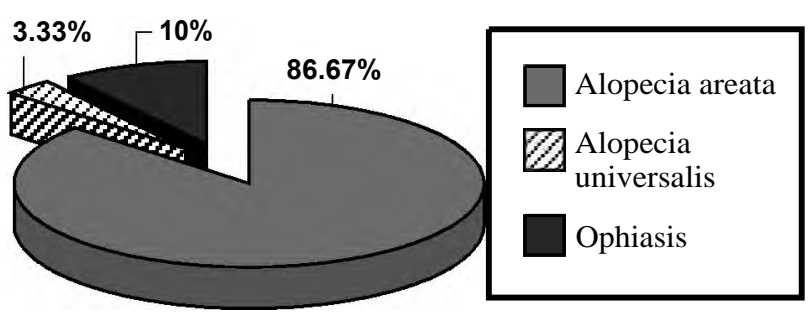

Fig. (2): Types of alopecia.

Out of 30 patients with TC, 20 patients $(66.67 \%)$ had scaly type, 5 patients (16.67\%) had black dot, 4 patient $(13.33 \%)$ had kerion Celsi and only one patient (3.33\%) had favus Fig. (3).

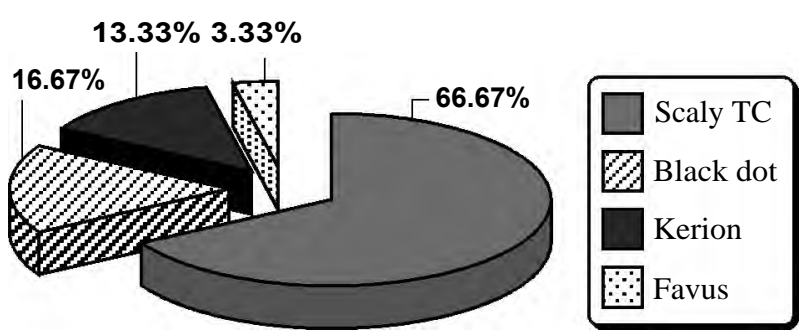

Fig. (3): Types of tinea capitis.

Alopecia group included 13 males (43.33\%) and 17 females $(56.67 \%)$. Their ages ranged from 2-12 years with mean \pm SD $6.70 \pm 3.54$ years. TC group included 22 males $(73.33 \%$ ) and 8 females (26.67\%). Their ages ranged from 1-12 years with mean \pm SD $6.42 \pm 3.37$ years Fig. (4).

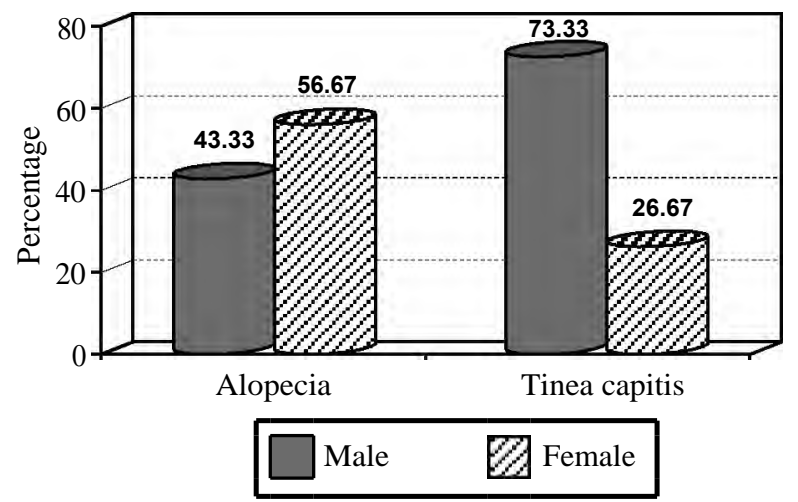

Fig. (4): Comparison between alopecia and tinea capitis regarding gender.
Duration of alopecia lesions ranged from 2-12 weeks with mean \pm SD $4.43 \pm 2.49$ weeks. The number of patients presented by one lesion was 26 patients $(86.67 \%)$ while patients presented by two lesions were 4 (13.33\%). As regards the size of the lesions, mean \pm SD was $(5.50 \pm 4.73) X(4.13 \pm$ 2.60) cm Fig. (5).

Duration of TC lesions ranged from 2-12 weeks with mean \pm SD $4.70 \pm 2.59$ weeks. The number of patients presented by one lesion was 29 patients (96.67\%) while only one patient presented by two lesions (3.33\%). Regarding the size of the lesions, mean \pm SD was $(4.43 \pm 1.29) X(4.06 \pm 1.10) \mathrm{cm}$ Fig. (5).

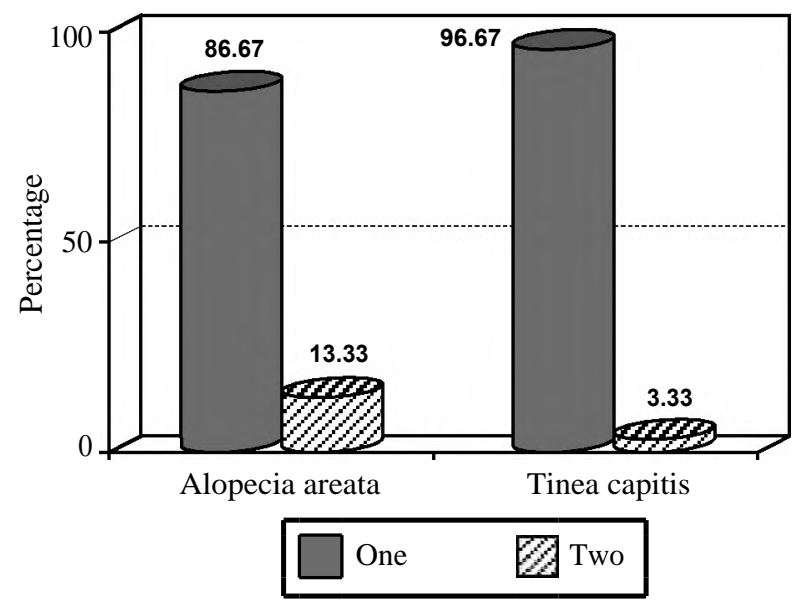

Fig. (5): Comparison between alopecia and tinea capitis regarding number of lesions.

Two patients $(6.67 \%)$ had AA in the frontal area, 3 patients $(10 \%)$ in the parietal area, 20 patients $(66.67 \%)$ in the vertex, 4 patients $(13.33 \%)$ in the occipital area and only one patient (3.33\%) involving the whole scalp. The shape of lesion differed from rounded in 19 patients $(63.33 \%)$, oval in 2 patients $(6.67 \%)$, irregular in 8 patients (26.67\%) and involving the whole scalp in only one patient (3.33\%) Figs. (6,7).

Five patients $(16.67 \%)$ had TC in the frontal area, 4 patients $(13.33 \%)$ in the parietal area, 18 patients $(60 \%)$ in the vertex and 3 patients $(10 \%)$ in the occipital area. The shape of lesions differed from rounded in 24 patients $(80 \%)$, oval in one patient $(3.33 \%)$, triangular in 2 patients $(6.67 \%)$ and irregular in 3 patients (10\%) Figs. $(6,7)$.

In the AA, the associated manifestation (itching, scaling and erythema) were absent in all patients $(100 \%)$, while in TC, itching present in 26 patients $(86.67 \%)$, scaling in 21 patients $(70 \%)$ and erythema in 7 patients (23.33\%) Fig. (8). 


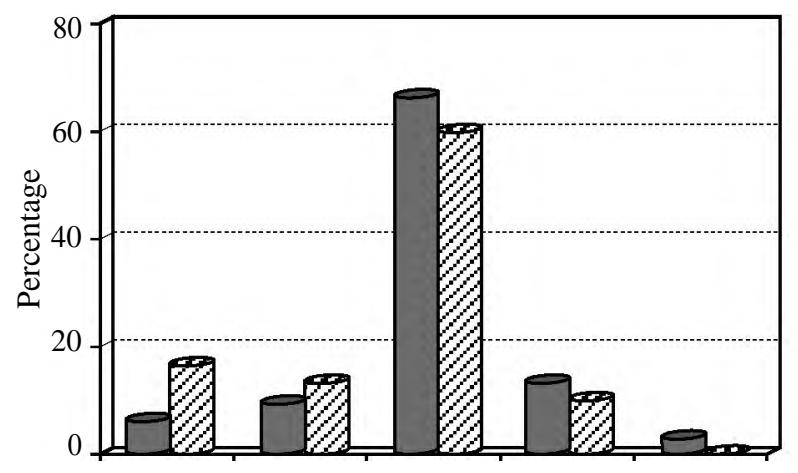

Frontal Parietal Vertex Occiput Whole

Site of lesions

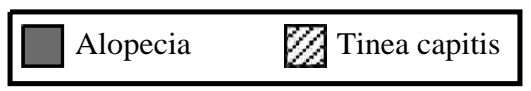

Fig. (6): Comparison between alopecia and tinea capitis regarding site of lesions.

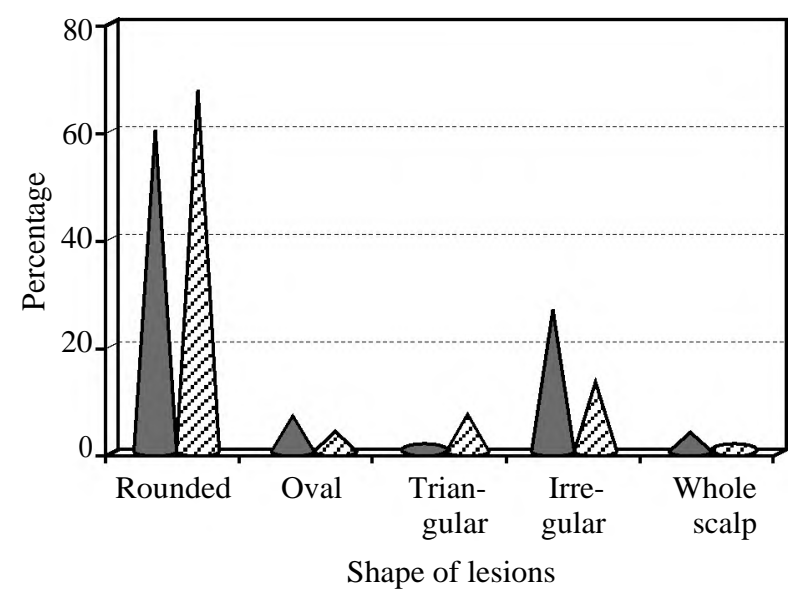

$\square$ Alopecia $\quad 2$ Tinea capitis

Fig. (7): Comparison between alopecia and tinea capitis regarding shape of lesions.

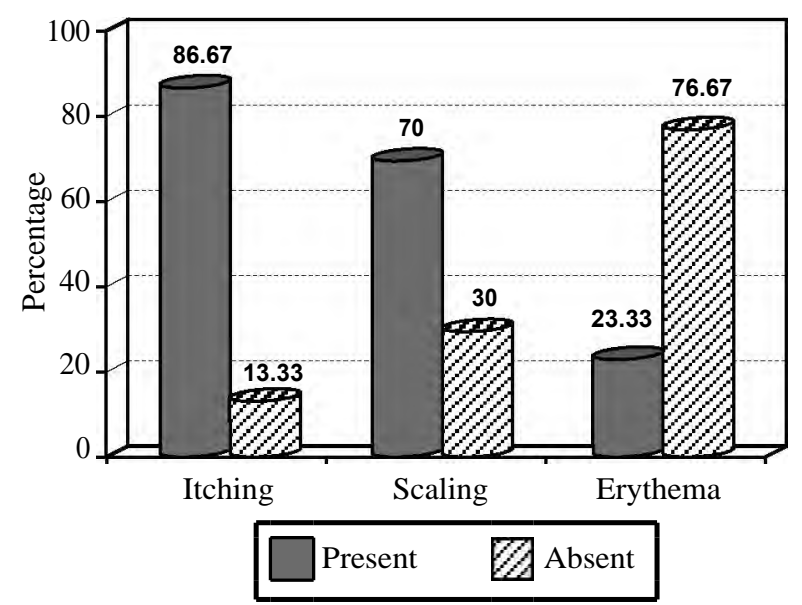

Fig. (8): Associated manifestations in tinea capitis.

The lesions of patchy hair loss in all patients were diagnosed according to the clinical findings into TC in 26 patients $(43.33 \%)$, AA in 29 patients
$(48.33 \%)$ and (TC or AA) in 5 patients $(8.34 \%)$ Fig. (9).

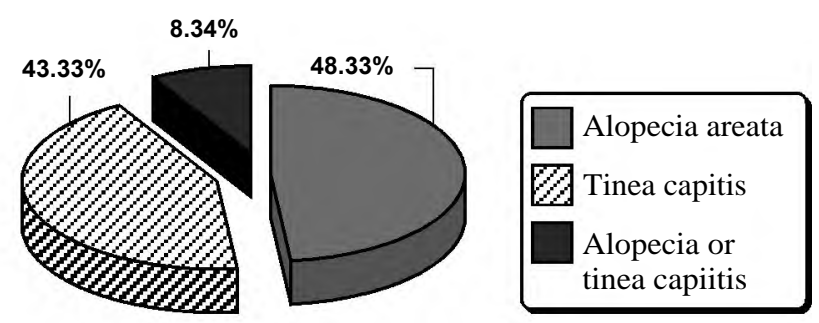

Fig. (9): Clinical diagnoses of the lesions.

\section{II- Laboratory results:}

Direct microscopic examination of the collected specimens from the lesions of patchy hair loss after being mounted by $\mathrm{KOH} 10 \%$ was done for all patients and revealed that all cases of AA $(100 \%)$ gave negative results, 22 patients $(73.33 \%)$ of TC had ectothrix, 5 patients $(16.67 \%)$ had endothrix result while 3 patients $(10 \%)$ had negative results Fig. (10).

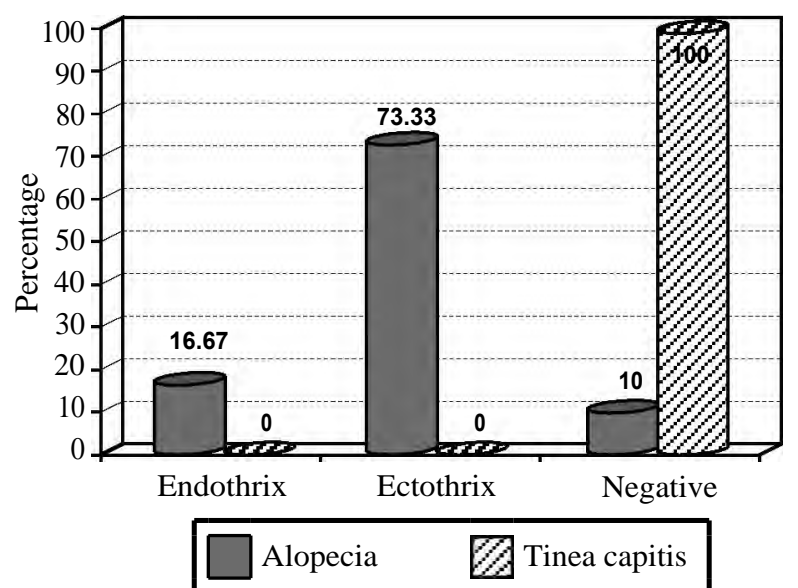

Fig. (10): Potassium hydroxide microscopic examination of alopecia and tinea capitis.

Fungal culture was done for all cases to confirm the clinical diagnosis and revealed that all cases of AA (100\%) gave negative result while dermatophyte isolated from TC cases were $M$. canis in 20 patients $(66.66 \%)$, T. violeceum in 6 patients (20\%), T. verrucosum in 2 patients $(6.67 \%)$ and M. auodinii in 2 patients (6.67\%) Fig. (11).

\section{III- Dermoscopic results:}

Out of 30 patients with TC, short broken hair was reported in 21 patients (70\%), black dots in 21 patients $(70 \%)$, comma shaped hairs in 24 patients $(80 \%)$, cork screw hairs in 12 patients (40\%), zigzag shaped hairs with bar code appearance in 20 patients $(66.67 \%)$ and white scales in all patients (100\%) Fig. (12). 
Out of 30 patient with alopecia, black dots were found in 24 patients (80\%), yellow dots in 15 patients $(50 \%)$, exclamation marks in 16 patients $(53.33 \%)$, white hairs in 13 patients
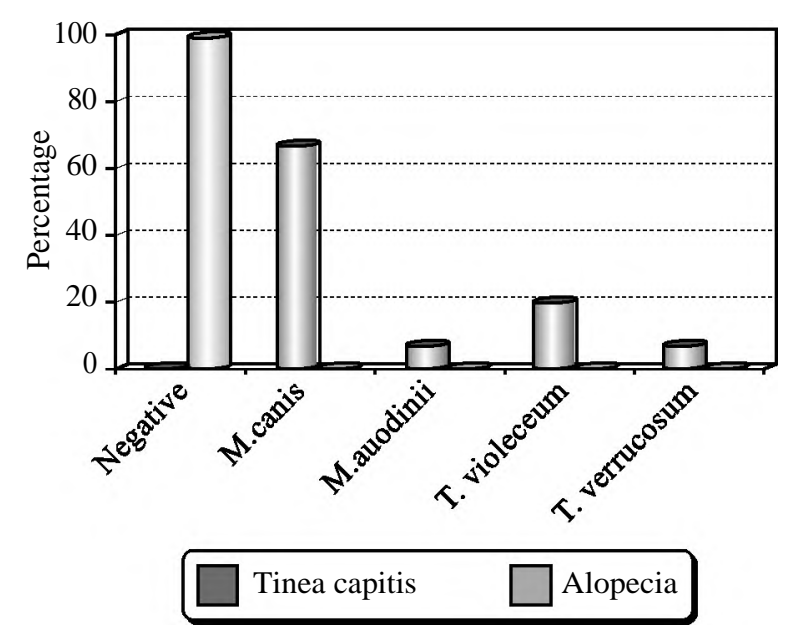

Fig. (11): Culture results of alopecia and tinea capitis.

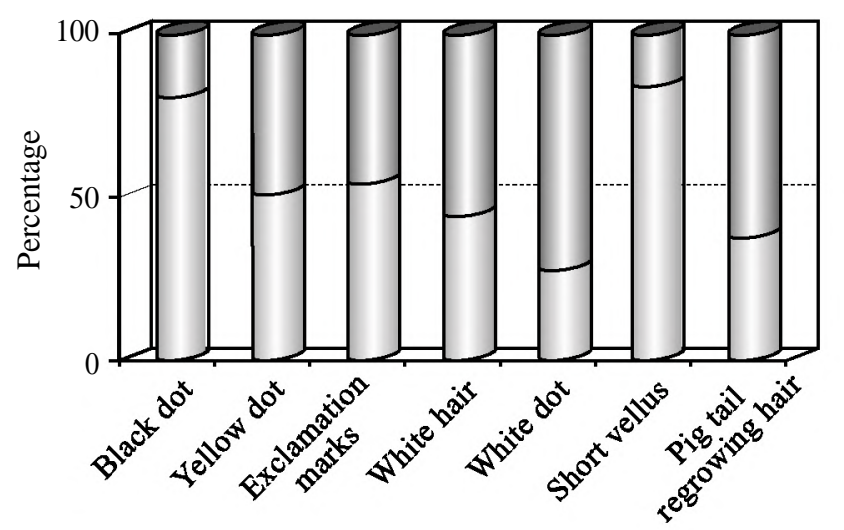

(43.33\%), white dots in 8 patients $(26.67 \%)$, short vellus hairs in 25 patients $(83.33 \%)$ while pig tail re-growing hairs in 11 patients $(36.67 \%)$ Fig. (13).

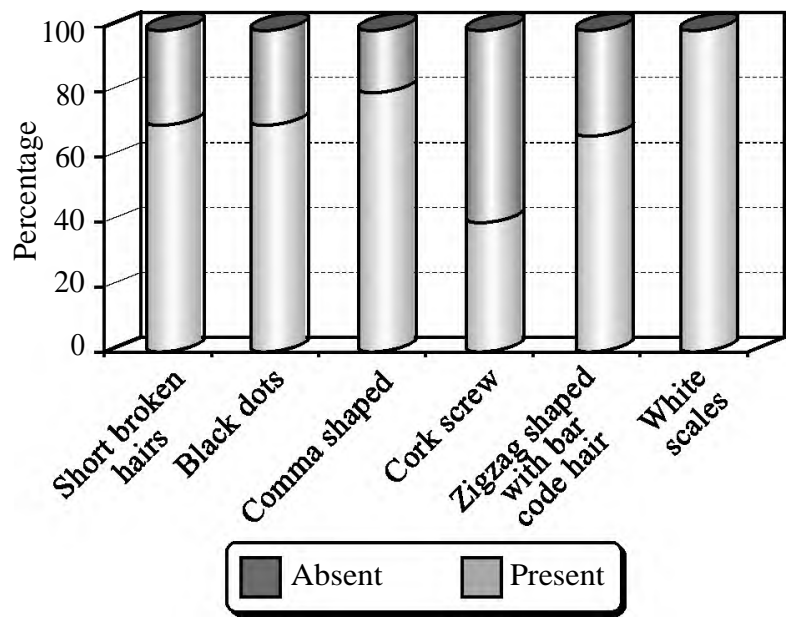

Fig. (12): Dermoscopic features of tinea capitis.

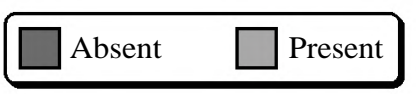

Fig. (13): Dermoscopic features of alopecia.
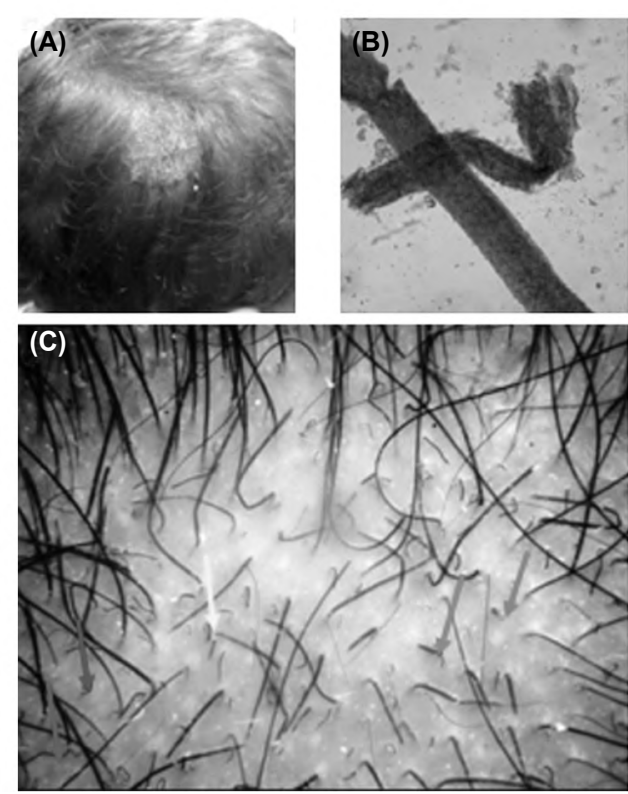

(D)
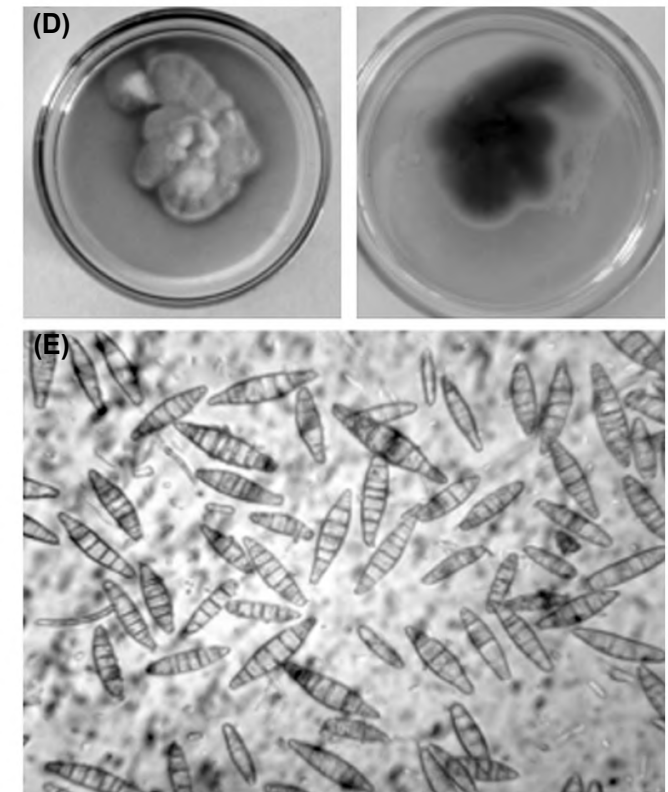

Fig. (14): Tinea capitis (non inflammatory scaly type caused by M. canis): (A) Macroscopic view, (B) KOH preparation for hair (ectothrix), (C) Dermoscopic view shows cork screw hair (red arrow), comma shaped hair (yellow arrow), zigzag hair (blue arrow), short broken hair (green arrow) and black dot (orange arrow), (D) Culture macroscopic view shows cottony white colony with yellowish periphery and the reverse color is yellow to brown, and (E) Culture microscopic view shows numerous spindle shaped macroconidia with septa. 

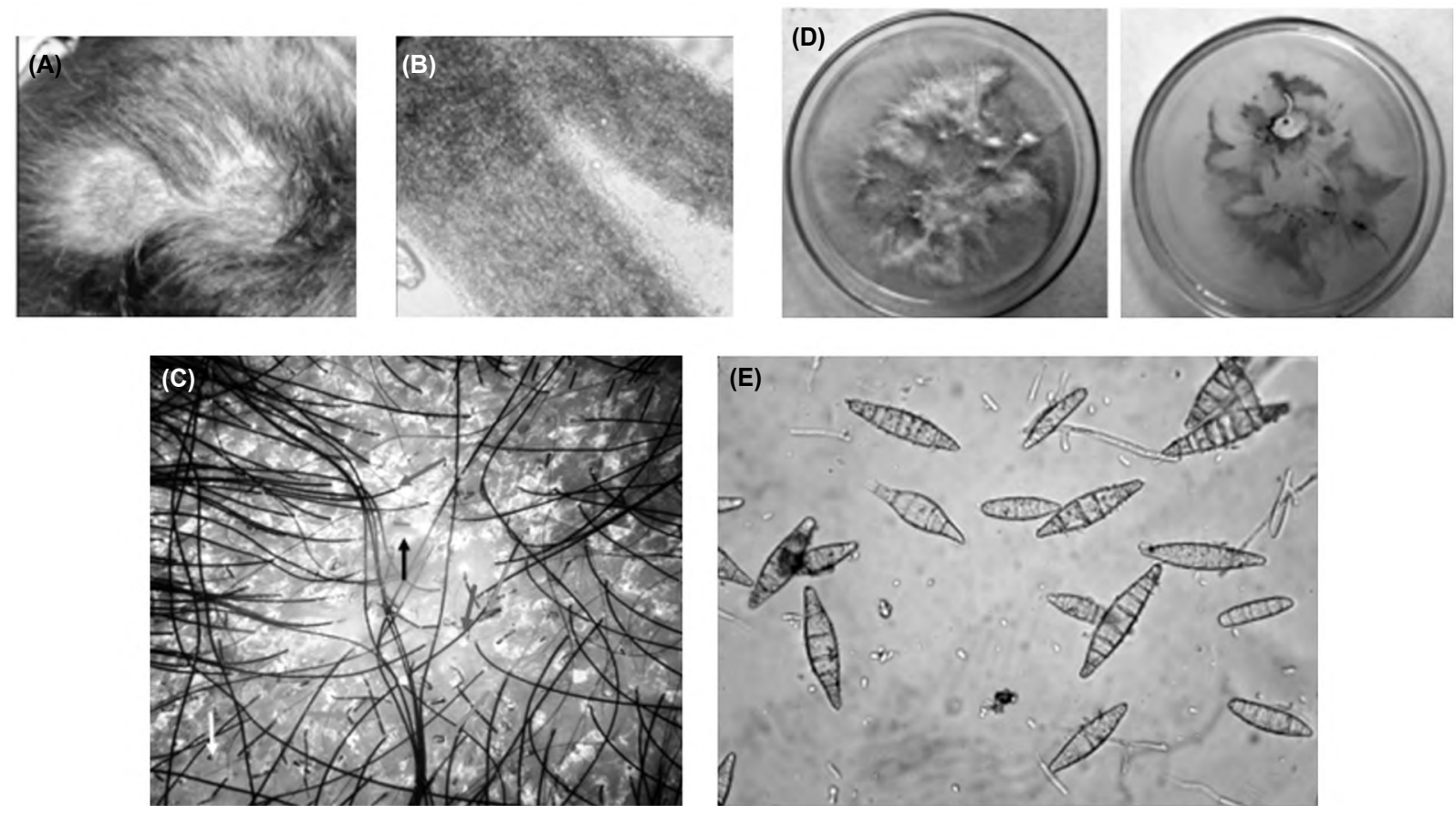

Fig. (15): Tinea capitis (non inflammatory scaly type caused by M. canis): (A) Macroscopic view, (B) KOH preparation for hair (ectothrix), (C) Dermoscopic view shows cork screw hair (red arrow), comma shaped hair (orange arrow), zigzag hair (green arrow), white scale (blue arrow), black dot (yellow arrow) and short broken hair (black arrow), (D) Culture macroscopic view shows cottony white colony with yellowish periphery and reverse color is yellow to brown, and (E) Culture microscopic view shows numerous spindle shaped macroconidia with septa.
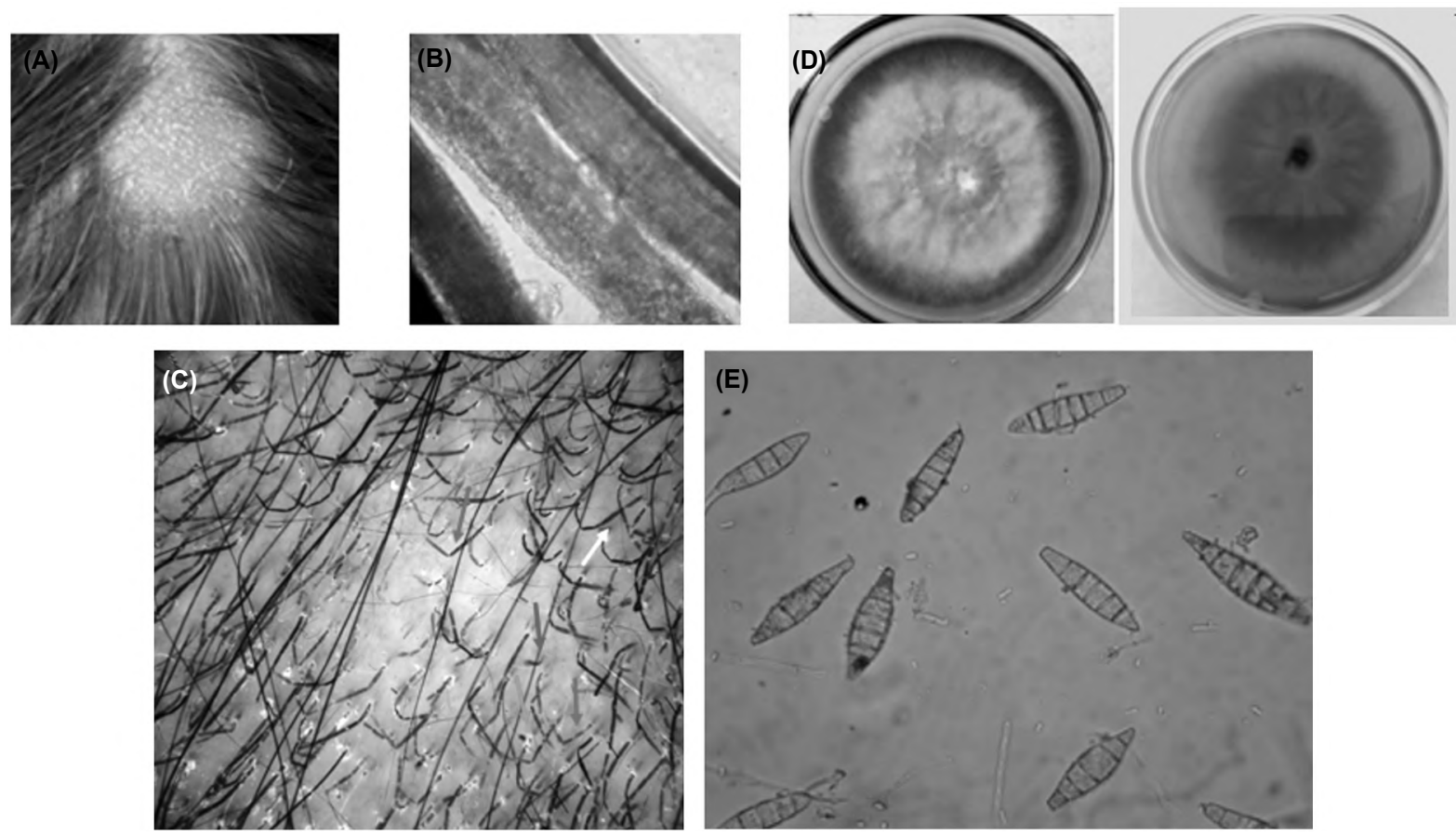

Fig. (16): Tinea capitis (non inflammatory scaly type caused by M. canis): (A) Macroscopic view, (B) KOH preparation for hair (ectothrix), (C) Dermoscopic view shows comma shaped hair (red arrow), zigzag hair with bar code appearance (blue arrow), black dot (green arrow) and short broken hair (yellow arrow), (D) Culture macroscopic view shows cottony white colony with yellowish periphery and the reverse color is yellow to brown, and (E) Culture microscopic view shows numerous spindle shaped macroconidia with septa. 

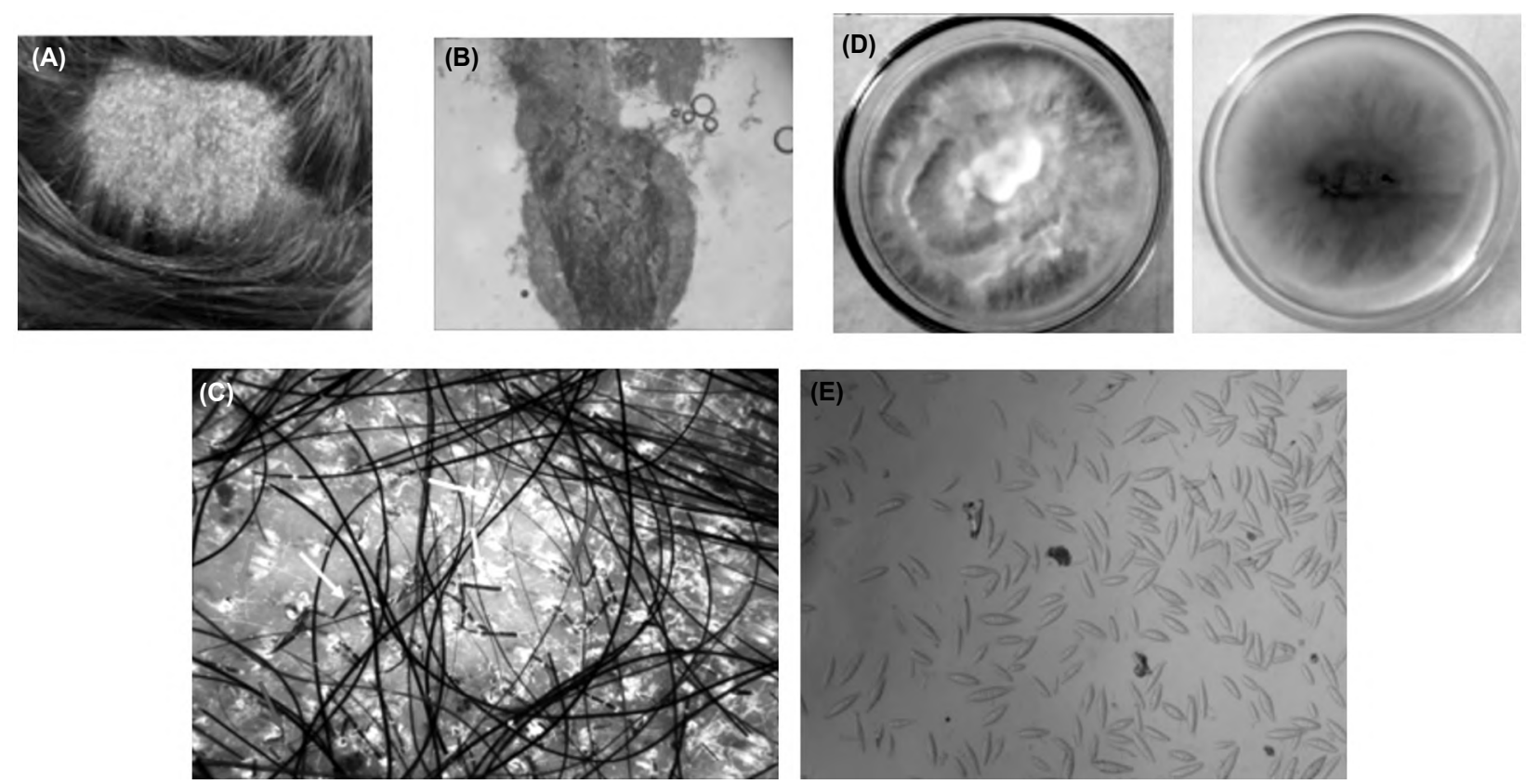

Fig. (17): Tinea capitis (non inflammatory scaly type caused by M. canis): (A) Macroscopic view, (B) KOH preparation for hair (ectothrix), (C) Dermoscopic view shows comma shaped hair (red arrow), zigzag hair (yellow arrow), black dot (green arrow), short broken hair (blue arrow) and white scale (white arrow), (D) Culture macroscopic view shows cottony white colony with yellowish periphery and the reverse color is yellow to brown, and (E) Culture microscopic view shows numerous spindle shaped macroconidia with septa.
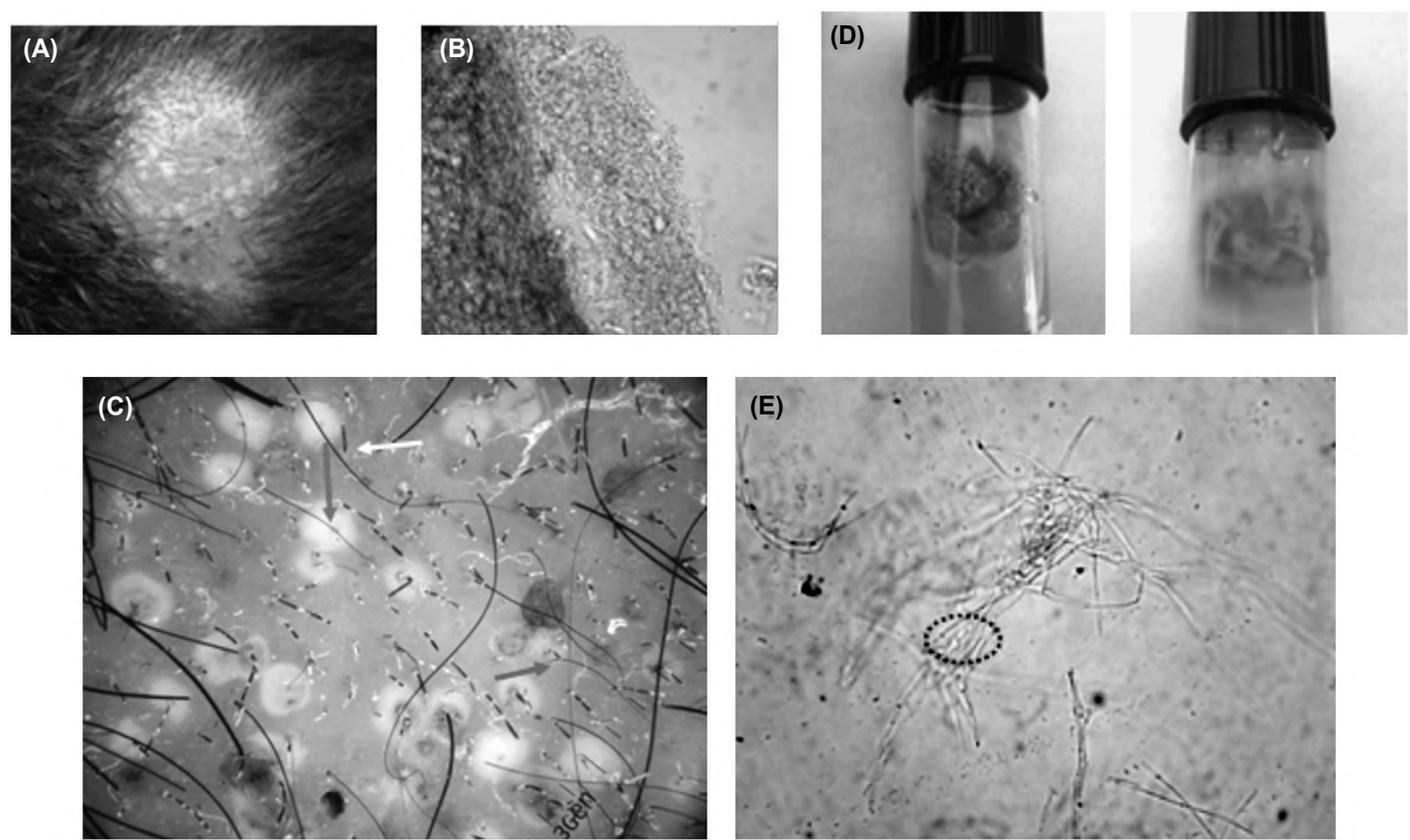

Fig. (18): Tinea capitis (inflammatory type-kerion caused by T. verrucosum): (A) Macroscopic view, (B) KOH preparation for hair (ectothrix), (C) Dermoscopic view shows comma shaped hair (red arrow), zigzag hair with bar code appearance (orange arrow), black dot (green arrow), short broken hair (yellow arrow) and pustule (blue arrow), (D) Culture macroscopic view shows white cottony, heaped and verrucosed, and (E) Culture microscopic view shows thin hyphae and chain of chlamydospore (red circle). 

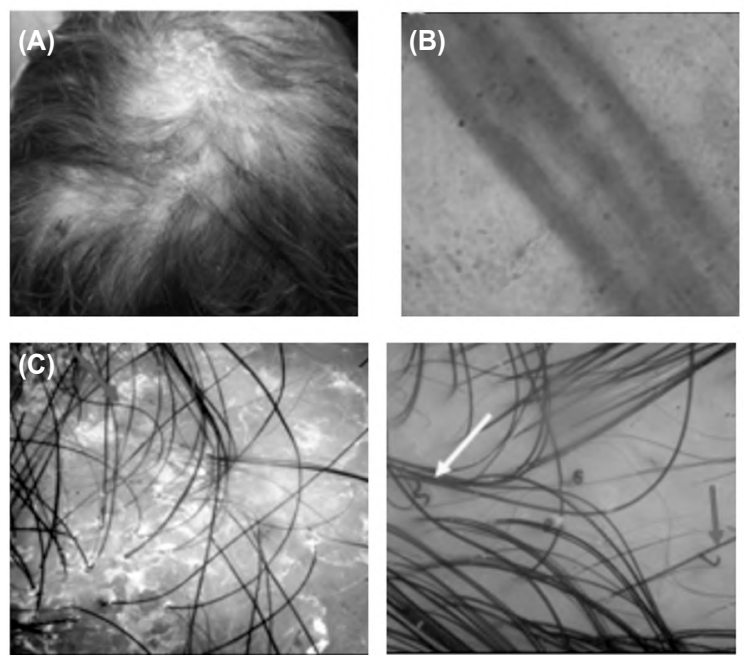
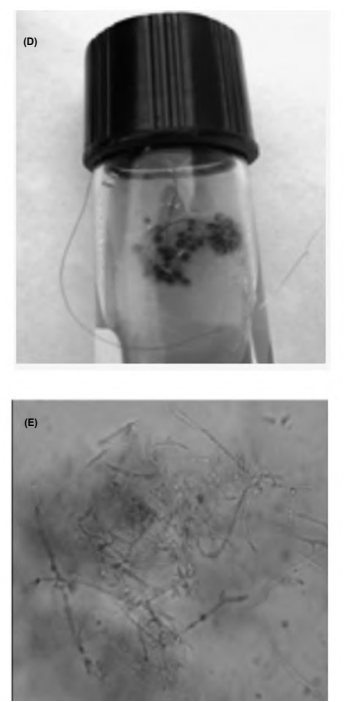
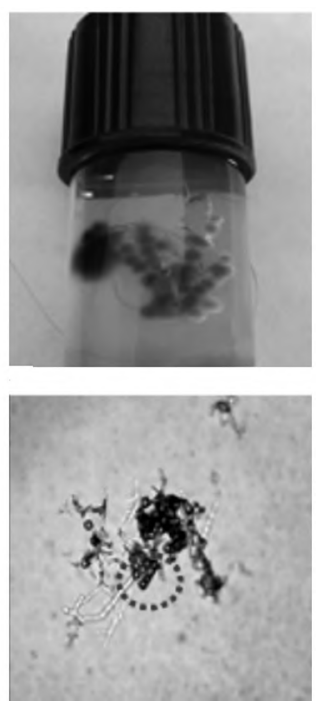

Fig. (19): Tinea capitis (inflammatory type - favus caused by T. violaceum): (A) Macroscopic view, (B) KOH preparation for hair (negative), (C) Dermoscopic view shows comma shaped hair (blue arrow), cork screw hair (yellow arrow) and scale (red arrow), (D) Culture macroscopic view shows diffuse, waxy colony and its surface and reverse color is deep violet, and (E) Culture microscopic view shows bizarre shaped hyphae and chlamydospore in old culture (red circle).
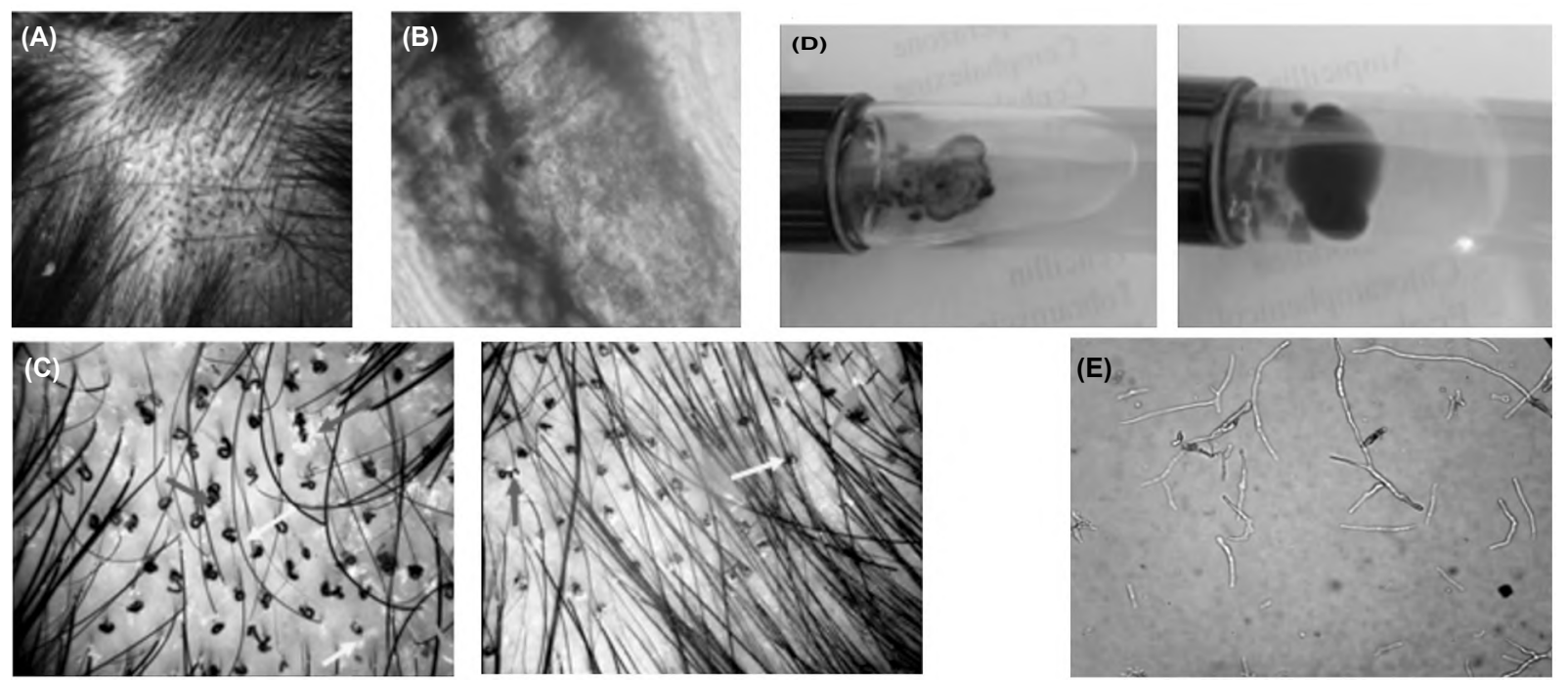

Fig. (20): Tinea capitis (non inflammatory black dot type caused by T. violaceum): (A) Macroscopic view, (B) KOH preparation for hair (endothrix), (C) Dermoscopic view shows comma shaped hair (yellow arrow), cork screw hair (red arrow), scale (orange arrow) and black dot (green arrow), (D) Culture macroscopic view shows that the surface and reverse color of the colony is deep violet, and (E) Culture microscopic view shows branched septated hyphae.
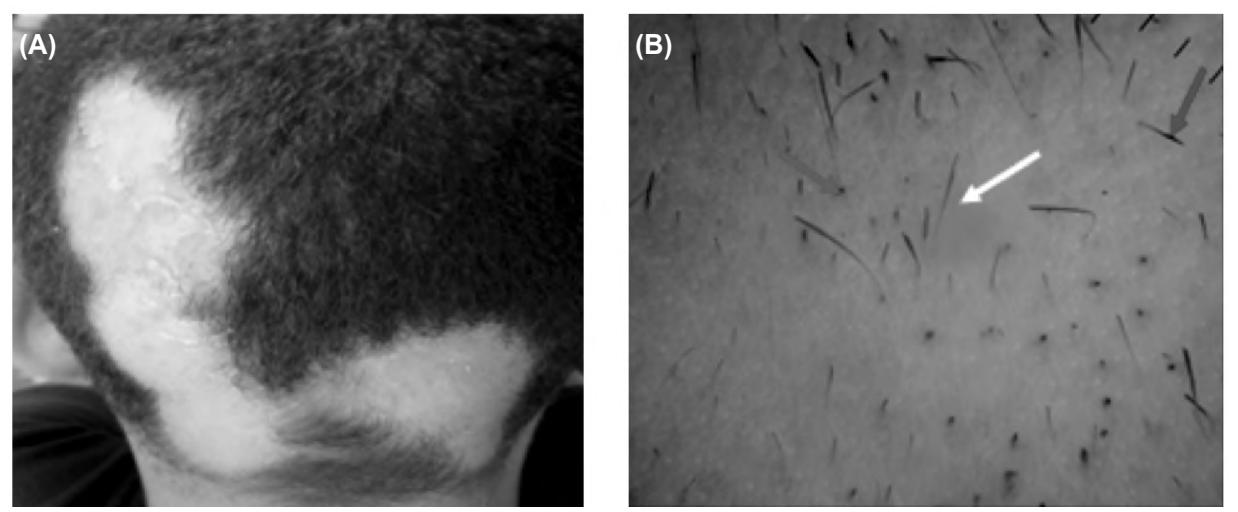

Fig. (21): Alopecia areata: (A) Macroscopic view, and (B) Dermoscopic view shows exclamation mark (red arrow), black dot (blue arrow) and vellus hair (yellow arrow). 

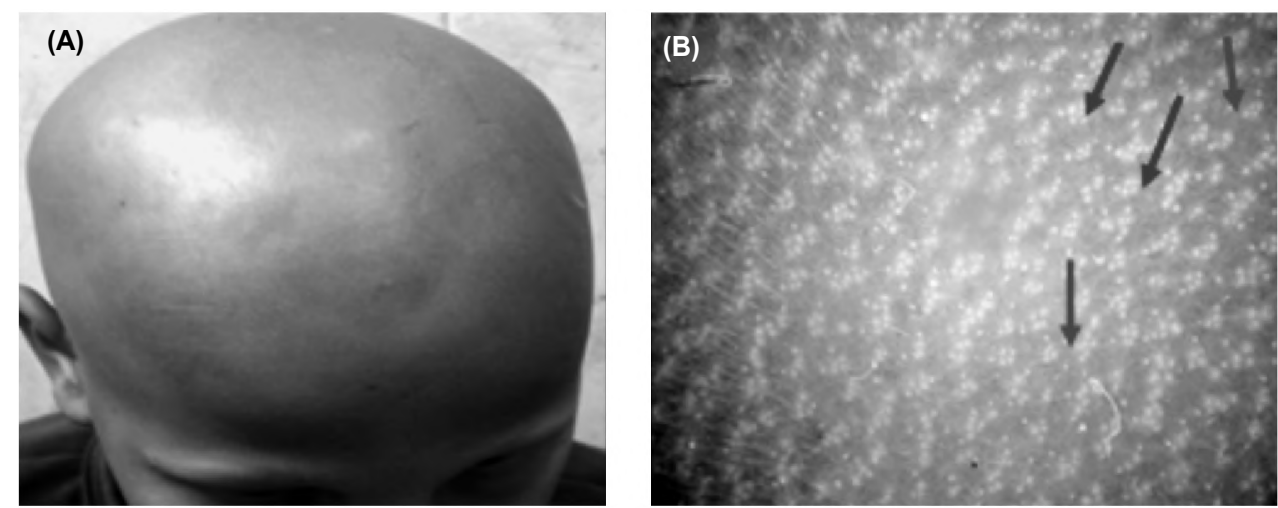

Fig. (22): Alopecia universalis: (A) Macroscopic view, and (B) Dermoscopic view shows isolated pin point white dot between follicular unit (blue arrow) and white dots in groups of two or three surrounded by white circular halo representing empty follicle (red arrow).
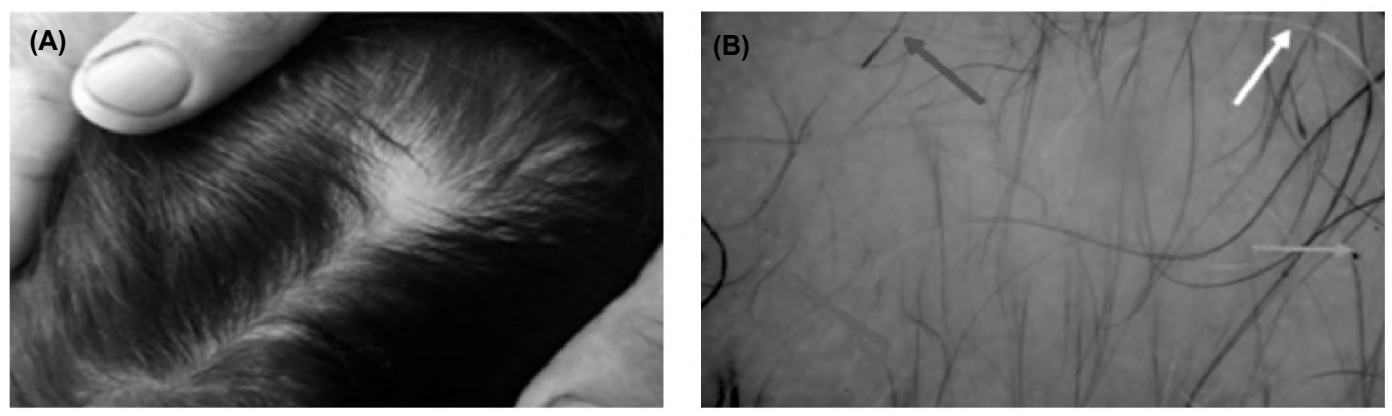

Fig. (23): Alopecia areata: (A) Macroscopic view, and (B) Dermoscopic view shows exclamation mark (red arrow), black dot (green arrow), vellus hair (blue arrow) and white hair (yellow arrow).
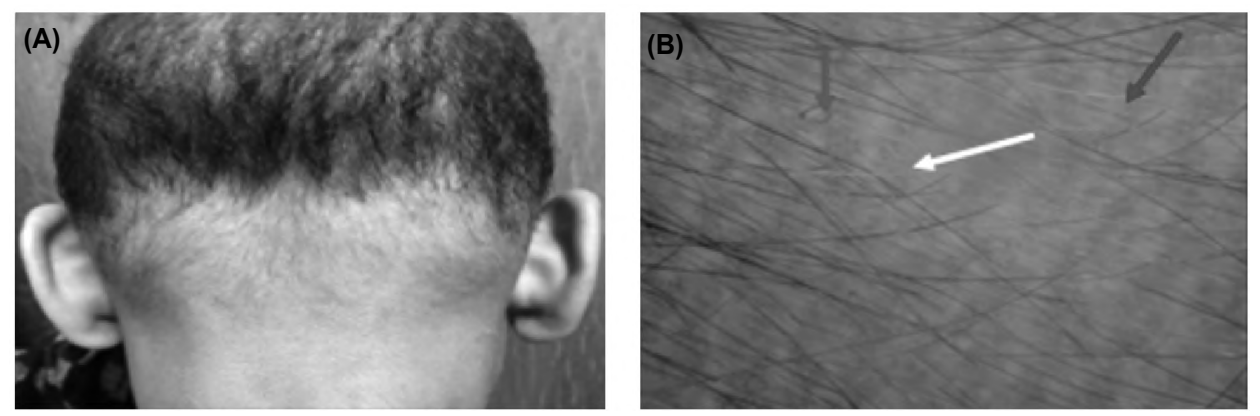

Fig. (24): Ophiasis: (A) Macroscopic view, and (B) Dermoscopic view shows pig tail re-growing hair (blue arrow), white hair (red arrow) and yellow dot (yellow arrow).
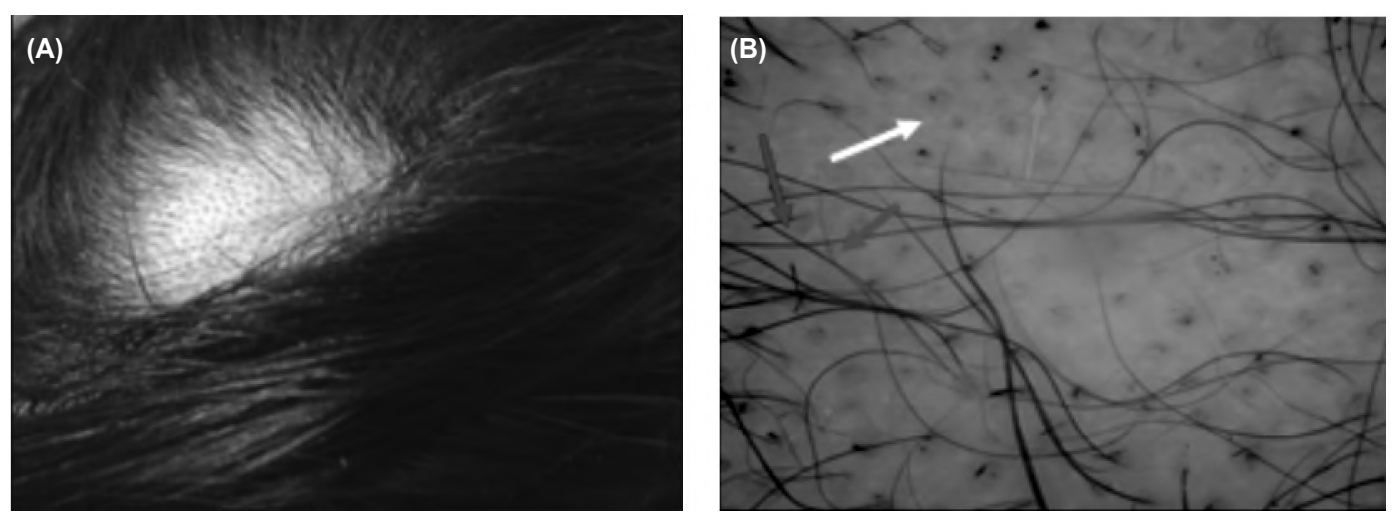

Fig. (25): Alopecia areata: (A) Macroscopic view, and (B) Dermoscopic view shows exclamation mark hair (red arrow), black dot (green arrow), yellow dot (yellow arrow) and vellus hair (blue arrow). 

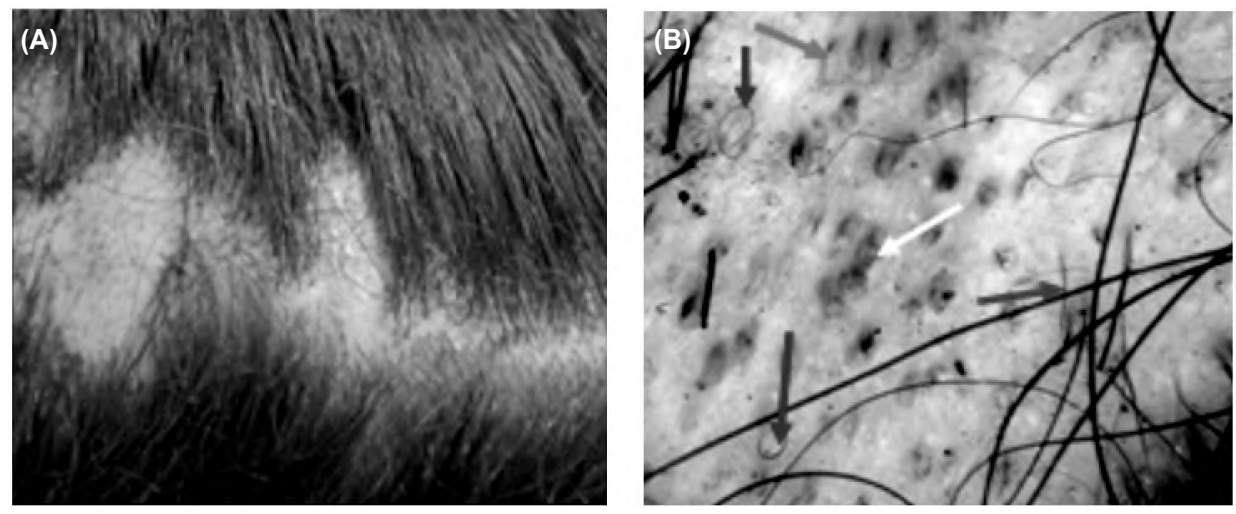

Fig. (26): Alopecia areata: (A) Macroscopic view, and (B) Dermoscopic view shows pig tail re-growing hair (red arrow), black dot (green arrow), yellow dot (yellow arrow) and vellus hair (blue arrow).
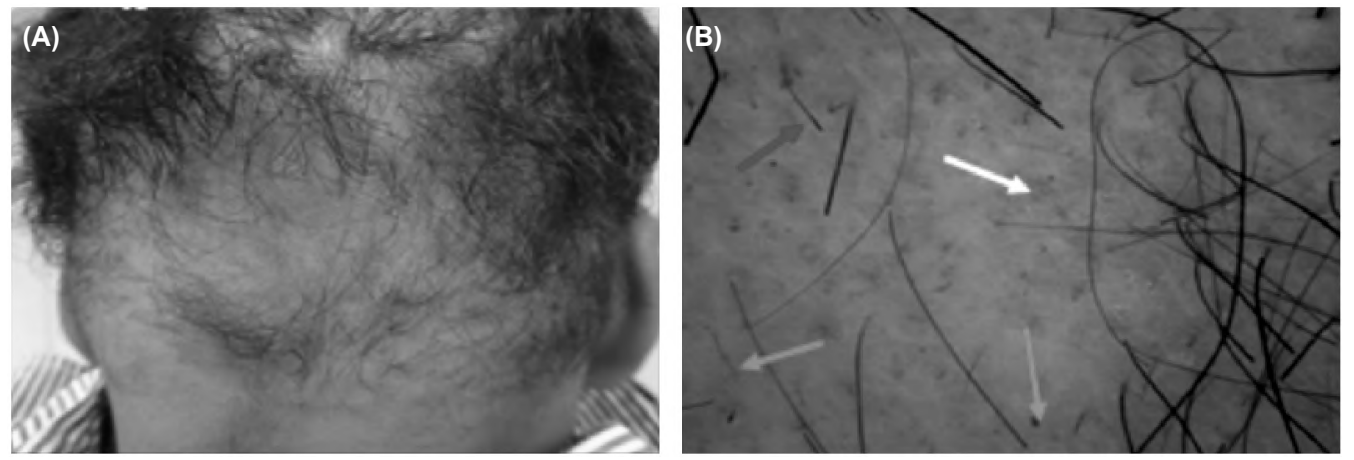

Fig. (27): Ophiasis: (A) Macroscopic view, and (B) Dermoscopic view shows exclamation mark hair (red arrow), black dot (green arrow), yellow dot (yellow arrow) and vellus hair (orange arrow).
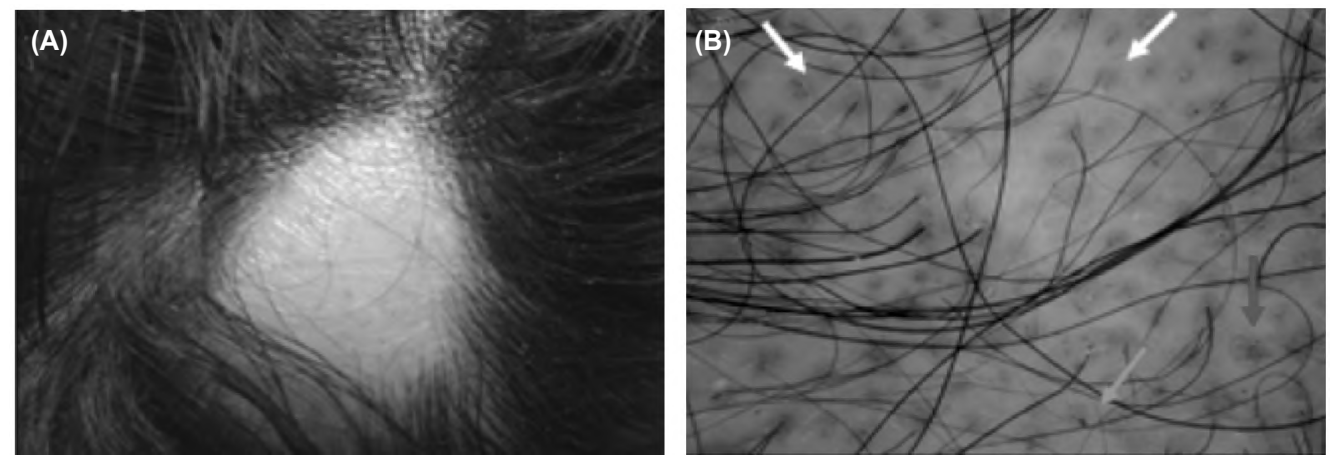

Fig. (28): Alopecia areata: (A) Macroscopic view, and (B) Dermoscopic view shows pig tail re-growing hair (red arrow), black dot (green arrow) and yellow dot (yellow arrow).
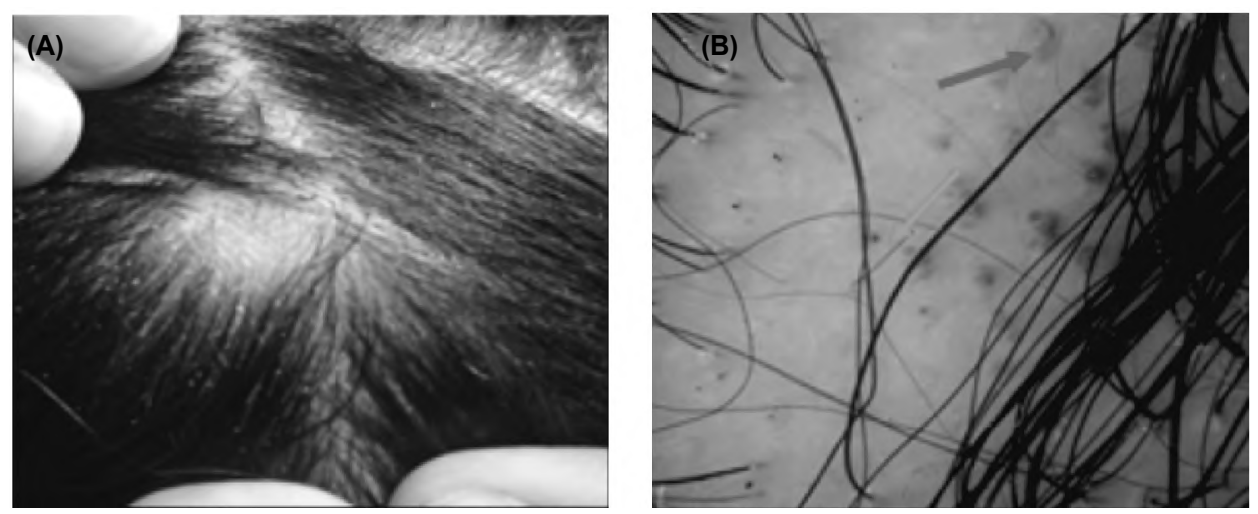

Fig. (29): Alopecia areata: (A) Macroscopic view, and (B) Dermoscopic view shows black dot (green arrow) and yellow dot (red arrow). 


\section{Discussion}

Tinea capitis and alopecia areata are considered the most common causes of hairless patches of the scalp in pediatrics [19]. TC may have the same clinical appearance of AA, so dermoscopy has recently become a useful diagnostic tool for both AA and TC, especially in doubtful cases, as laboratory investigations like fungal culture or biopsy may takes several weeks while dermoscopy is considered a rapid and non invasive method of diagnosis of hair disorders [15].

The studies regarding dermoscopic findings of patients with TC were few and included few patients [20]. They stated that, comma hairs and corkscrew hairs are two hair shaft abnormalities frequently observed in TC. In the present study, we found by dermoscopic examination of TC patients, comma shaped hairs, zigzag shaped hairs, corck screw hairs, black dots and short broken hairs. These results were in agreement with Ekiz et al., [21].

In the present study under dermoscopic examination, comma shaped hairs were seen in $80 \%$ of patients. This finding goes in accordance with those of Slowinska et al., [22] and Hughes et al., 2011 [23], who detected comma shaped hairs in $100 \%$ of their patients. Ekiz et al., 2014 [21] reported comma shaped hairs in $66.7 \%$ of their patients. Comma hairs, which are slightly curved and fractured hair shafts, are associated with ectothrix and endothrix type fungal invasion. The comma hair is believed to be probably shaped as a result of subsequent cracking and bending of a hair shaft filled with hyphae [22]

In the current study under dermoscopic examination, zigzag shaped hairs were seen in $66.67 \%$ of patients, and corkscrew hairs in $40 \%$ of patients. These findings were detected in Hughes et al., [23] study. They reported corkscrew hairs in $67 \%$ of their patients. Ekiz et al., 2014 [21] reported it in $80 \%$ of their patients. The zigzag shaped hairs or corkscrew hairs seems to be variations of the comma hairs, manifesting in black patients [23] They did not explain this finding.

Short broken hairs were observed in $70 \%$ of the studied TC patients under dermoscopic examination. This finding was detected in other studies as Mapelliet et al., [20] and Ekiz et al., 2014 [21] in $100 \%$ of studied patients. Short broken hairs may not be specific dermoscopic finding of TC, but may be a sign of severity of the disease, as all cases of TC were not treated before the study.
Black dots reported in our study under dermoscopic examination in $70 \%$ of TC patients. This finding was detected in Sandoval et al., 2010 study [24]. Black dots are remnants of broken hairs or dystrophic hairs [9].

Hughes et al., [23] stated that comma shaped hairs and corkscrew hairs were detected in zoophilic infection. They also stated that comma hairs could be observed on both Microspore and Tricophyton species, but corkscrew hairs were observed only on T. soudanense infection. Mapelliet et al., [20] performed their study on three black children. All cases had a diagnosis of $\mathrm{T}$. violeceum and large number of comma, broken and dystrophic hairs and no corkscrew hairs were observed in trichoscopic findings.

Ekiz et al., 2014 [21] reported that zoophilic fungi such as $\mathrm{T}$. verrucosum and $\mathrm{M}$. canis were isolated. In the present study, T. violaeceum (20\%), M. canis $(66.66 \%)$, T.verrucosum $(6.67 \%)$ and $\mathrm{M}$. auodinii $(6.67 \%)$ were isolated. The differences between our study and these studies may be due to the fact that farming is common and the socioeconomic status is low in many regions of our country.

The most common dermoscopic features in this study were short broken hairs (70\%), black dots (70\%), comma shaped hairs (80\%), corkscrew hairs $(40 \%)$ and zigzag shaped hairs in (66.67\%). Comma shaped hairs, zigzag shaped hairs or corkscrew hairs are characteristic dermoscopic features of TC. Black dots and short broken or dystrophic hairs are non specific to TC, as they can be observed also in AA, trichotillomania, but they could be used as signs of severity of TC.

In dermoscopic examination of patients with TC, broken and dystrophic hairs, zigzag shaped hairs or corkscrew hairs and comma hairs were reported in other studies [21-23]. However, unlike Huges et al., [23], in the present study, corkscrew hairs were detected in patients with T. violeceum and M. canis infection.

Trichophyton schoenleinii is the most frequently identified causative organism in favus, although few cases have been attributed to $T$. violaceum

In the present study, we have only one patient with favus and the causative organism was $T$. violaceum. Ilkit, [25] reported that $\mathrm{T}$. violaceum infections can last for long periods without any evidence of inflammation, and that such infections can change to kerion type of infection. 
Although the studies regarding trichoscopic findings of patients with TC are rare [26], there are large scale studies in patients with AA [21]. Yellow dots, black dots, broken hairs, exclamation mark and short vellus hairs are considered as characteristic dermoscopic features in AA [21,27].

In the present study, yellow dots are detected in $50 \%$ of studied patients. This finding was detected in other studies as Mane et al., 2011 [28] who reported yellow dots in $81.8 \%$ of their patients, Karadag and Güleç, [27] who reported them in 46.5\%, and Ekiz et al., [21] who reported them in $100 \%$. Ross et al., [6] mentioned that yellow dots are considered the most sensitive dermoscopic feature of AA. These are marked by distinctive array of yellow to yellow-pink, round or polycyclic dots that vary in size and are uniform in color. They are more easily observed using videodermoscopy than handheld dermoscopy [9]. The combination of large numbers of yellow dots and short re-growing hairs is a feature of AA incognita [13]. For the diagnosis of AA, other signs of AA should be taken into account, because isolated yellow dots may be seen in trichotillomania, hypotrichosis simplex and even TC [29]. Rudinicka et al., 2011 [13] mentioned that yellow dots occur in long lasting AA, but in the present study, yellow dots observed in cases with short duration. This may be due to the young age of present patients.

In the current study, under dermoscopic examination exclamation mark hairs was detected in $53.33 \%$ of AA patients. Other studies reported exclamation mark hairs in less percentages ranged from $12.1 \%$ to $40 \%$ [9,27,28] " "Tapering hair"; this term is preferred over "exclamation mark hair" because the affected hair is not typical exclamatory mark in shape. It occurs due to the narrowing of hair shafts toward the follicles which is more readily perceived using dermoscopy than by naked eye [9]. In the present study we believe that, tapering hairs are diagnostic feature of AA. This goes in agreement with other studies $[\mathbf{2 1 , 2 7 , 3 0 ]}$. It is a sign of active AA, as it was seen in the active cases of $\mathrm{AA}$ at the periphery of the lesions.

In the present study under dermoscopic examination, black dots detected in $80 \%$ of AA patients. Mane et al., [28] recorded black dots in $67.7 \%$ of their AA patients, while Rudinicka et al., 2011 [13] and Karadag and Güleç, [27] recorded them in only $27.1 \%$ of their AA patients. Black dots as remnants of exclamation mark hairs or broken hairs. They occur when hair shaft fractured before emerging from the scalp. They provide a sensitive marker for disease activity as well as severity of AA [9]
The present study showed that black dots were one of the most important dermoscopic finding and can be used as a sensitive feature of AA only if associated with other specific dermoscopic features of AA as yellow dots, tapering hairs or short vellus hairs. As in the present study, black dots were detected also in cases of TC.

In the present study under dermoscopic examination, short vellus hairs were detected in $83.33 \%$ of AA patients. Other studies reported short vellus hairs in less percentages ranging from $40.9-50 \%$ of the studied patients of AA [21,27,28]. Lacarrubba et al., [31] mentioned that short vellus hairs were seen as new, thin, and un pigmented hairs within the patch. They may or may not be detectable clinically. Inui et al., 2008 [9] showed that short vellus hairs is also a diagnostic feature of AA, which can provide useful prognostic information (indicates the non-destructive nature of AA. They also mentioned that the appearance of clusters of short vellus hairs is a possible sign of spontaneous remission or adequate treatment, but in the present study it was a sign of spontaneous remission as the cases were not treated before the study.

In the current study under dermoscopic examination, pig tail re-growing hairs were reported in $36.67 \%$ of AA studied patients. This finding was detected in other studies as Karadag and Güleç, 2012 [27] had detect lower percentage (4.1\%) in their study. Pig tail re-growing hairs is not common in this study, but if present it is a diagnostic dermoscopic finding and is a possible sign of spontaneous remission of AA.

In the present study under dermoscopic examination, white hairs were detected in $43.33 \%$ of AA patients. El Taweel et al., 2014 [32] found that it is a diagnostic dermoscopic finding and a sign of spontaneous remission of AA.

In the present study under dermoscopic examination, white dots were detected in $26.67 \%$ of AA patients. Rudnickal et al., 2008 [14] found that white dots were considered as sites of targeted follicular destruction (fibrosis) which can be seen in long standing AA.

Some of the dermoscopic features can be used to predict the activity and severity of AA. Tapering hair is considered as a marker of disease activity and known to reflect exacerbation of the disease. These dermoscopic findings will be helpful for management of patients with hair disorders. Yellow dots and short vellus hairs enable AA to be screened from other hair loss disorders. Abundant numbers of the yellow dots seen in AA could differentiate 
it from trichotillomania which can have limited number of yellow dots. In addition, black dots, tapering hairs and broken hairs are specific for AA, not for trichotillomania. Single dermoscopic feature may not reliably diagnose AA [28]. Inui et al., 2008 [9] found that a combination of cadaverized hairs, exclamation mark hairs, broken hairs and yellow dots could sensitively detect difficult clinically diagnose types of AA like AA incognito. Broken hairs may be found in TC and trichotillomania.

So, the most common dermoscopic features weres short vellus hairs, black dots, exclamation marks, white hairs and pig tail re-growing hairs in percentages $83.33 \%, 80 \%, 53.33 \%, 50 \%, 43.33 \%$, and $36.67 \%$ respectively. The yellow dots, exclamation mark hairs and short vellus hairs are specific to AA. If they were not detected under dermoscopy, further clinical and histopathological examination will be required.

\section{Conclusion:}

Diagnosis of patchy hair loss in pediatric patients is often a matter of considerable debate among dermatologists. Dermoscopy can be viewed as rapid and non invasive tool to detect the cause of patchy hair loss. Like clinical dermatology, dermoscopy works parallel to the skin surface and perpendicular to the histological plane; like the histopathology, thus it allows the viewing of structures not discernible by the naked eye. This study included 60 patients (30 patients with tinea capitis and 30 patients with alopecia areata). They were examined clinically, lab investigations done as direct microscopic examination with $10 \% \mathrm{KOH}$ and fungal culture to confirm clinical diagnosis and dermoscopically, aiming at finding the different dermoscopic features of TC and AA. The most common dermoscopic features reported in TC were short broken hairs and black dots, but both of them non specific as they were detected in other conditions of hair loss. Comma shaped hairs, corkscrew hairs, and zigzag shaped hairs are the diagnostic dermoscopic features of TC. In AA patients, the most common dermoscopic features were black dots but it is not specific for AA, as it is found in other conditions as TC. But the specific feature was yellow dots, exclamation mark and short vellus hairs. From this study, we concluded that dermoscopy may represent a rapid and reliable confirmatory test. It can be used to differentiate between TC and AA by detecting their characteristic dermoscopic features. In TC, the characteristic findings are comma shaped hairs, zigzag hairs or corkscrew hairs which do not present in AA. In AA, the characteristic findings are yellow dots or exclamation marks which do not present in TC.

\section{Recommendations:}

Short-training-based dermoscopy will improve the confidence in the diagnosis of different hair diseases digital dermoscopy may be used in the near future as a computer-assisted diagnosis.

Tele-dermoscopy should be used among dermatologists to exchange difficult or interesting images and to help less-experienced dermatologists. The limitations of our study were the small number of patients and the absence of controls. A blinded study with a larger group of patients is needed to further define the role of dermoscopy in the clinical setting of tinea capitis and alopecia areata.

\section{References}

1- GHANNOUM M., ISHAM N., HAJJEH R., et al.: Tinea capitis in Cleveland: Survey of elementary school students. J. Am. Acad. Dermatol., 48: 189-93, 2003.

2- ELEWSKI B.E.: Tinea capitis: A current perspective. J. Am. Acad. Dermatol., 42: 1-20, 2000.

3- ALVAREZ M.S. and SILVERBERG N.B.: Tinea capitis. Cutis., 78: 189-96, 2006.

4- HAY R.J. and MOORE M.K.: Mycology. In: Burns T., Breathnach S., Cox N., et al. (eds.): Rook's Textbook of Dermatology. 7 th ed. Massachusetts: Blackwell Publishing Ch. 31, P. 1-101, 2004

5- ANDREWS M.D. and BURNS M.: Common tinea infections in children. Am. Fam. Physician, 77: 1415-20, 2008.

6- ROSS E.K., VINCENZI C. and TOSTI A.: Videodermatoscopy in the evaluation of hair and scalp disorders. J. Am. Acad. Dermatol., 55: 799-806, 2006.

7- PAUS R., OSLEN E.A. and MESSENGER A.G.: Hair Growth Disorders. In: Wolff K., Goldsmith A. and Katz S.I. (eds.): Fitzpatrick's Dermatology in General Medicine. New York, McGraw-Hill, USA, 7th ed., P. 763, 2008.

8- INUI S., NAKAJIMA T. and ITAMI S.: Dry dermoscopy in clinical treatment of alopecia areata. J. Dermatol., 34: 635-9, 2007.

9- INUI S., NAKAJIMA T., NAKAGAWA K., et al.: Clinical significance of dermoscopy in alopecia areata: Analysis of 300 cases. Int. J. Dermatol., 47: 688-93, 2008.

10- BRAUN R.P., RABINOVITZ H.S., OLIVIERO M., et al.: Dermoscopy of pigmented skin lesions. J. Am. Acad. Dermatol., 52: 109-21, 2005.

11-MICALI G., LACARRUBBA F., MASSIMINO D., et al.: Dermoscopy. alternative uses in daily clinical practice. J. Am. Acad. Dermatol., 64: 1135-46, 2011.

12- ZALAUDEK I., ARGENZIANO G., DiSTEFANI A., et al.: Dermoscopy in general dermatology. Dermatol., 212: 7-18, 2006.

13- RUDNICKA L., OLSZEWSKA M., RAKOWSKA A., et al.: Trichoscopy update 2011. J. Dermatol. Case Rep., 5: 82-8, 2011. 
14- RUDNICKA L., OLSZEWSKA M., RAKOWSKA A., et al.: Trichoscopy: A new method for diagnosing hair loss. J. Drugs Dermatol., 7: 651-4, 2008.

15- HALIASOS E.C., KERNER M., JAIMES-LOPEZ N., et al.: Dermoscopy for the pediatric dermatologist Part I: Dermoscopy of pediatric infectious and inflammatory skin lesions and hair disorders. Pediatr. Dermatol., 30: 163-71, 2013.

16- RAKOWSKA A.: Trichoscopy (hair and scalp videodermoscopy) in the healthy female. Method standardization and norms for measurable parameters. J. Dermatol. Case Rep., 3: 14-9, 2009.

17- RAKOWSKA A., SLOWINSKA M., KOWALSKAOLEDZKA E., et al.: Trichoscopy in genetic hair shaft abnormalities. J. Dermatol. Case Rep., 2: 14-20, 2008.

18- TOSTI A., WHITING D., IORIZZO M., et al.: The role of scalp dermatoscopy in the diagnosis of alopecia areata incognita. J. Am. Acad. Dermatol., 59: 64-7, 2008.

19- HILLMANN K. and BLUME-PEYTAVI U.: Diagnosis of hair disorders. Semin. Cutan. Med. Surg., 28 (1): 33 8, 2009.

20- MAPELLIET E.T., GUALANDRI L. and CERRI A.: Comma hairs in tinea capitis: A useful dermatoscopic sign for diagnosis of tinea capitis. Pediatr. Dermatol., 29: 223-4, 2012.

21- EKIZ O., SEN B.B., RIFAIOGLU E.N., et al.: Trichoscopy in paediatric patients with tinea capitis: A useful method to differentiate from alopecia areata. J. Eur. Acad. Dermatol. Venereol., 28 (9): 1255-8, 2014.

22- SLOWINSKA M., RUDNICKA L., SCHWARTZ R.A., et al.: Comma hairs: A dermatoscopic marker for tinea capitis: A rapid diagnostic method. J. Am. Acad. Dermatol., 59 (5 Suppl): 77-9, 2008.
23- HUGHES R., CHIAVERINI C., BAHADORAN P., et al.: Corkscrew hair: A new dermoscopic sign for diagnosis of tinea capitis in black children. Arch. Dermatol., 147: 355-6, 2011.

24- SANDOVAL A.B., ORTIZ J.A., RODRIGUEZ J.M., et al.: Dermoscopic pattern in tinea capitis. Rev. Iberoam. Micol., 27: 151-2, 2010.

25- ILKIT M.: Favus of the scalp: An overview and update. Mycopathologia, 170: 143-54, 2010.

26- VAZQUEZ-LOPEZ F., PALACIOS-GARCIA L. and ARGENZIANO G.: Dermoscopic corkscrew hairs dissolve after successful therapy of trichophyton violaceum tinea capitis: A case report. Aust. J. Dermatol., 53: 118-9, 2012.

27- KARADAG KOSE O. and GULEC A.T.: Clinical evaluation of alopecias using a handheld dermatoscope (in Press). J. Am. Acad. Dermatol., 67 (2): 206-14, 2012.

28- MANE M., NATH A.K. and THAPPA D.M.: Utility of dermoscopy in alopecia areata. Indian J. Dermatol., 56: 407-11, 2011.

29- INUI S.: Trichoscopy for common hair loss diseases: Algorithmic method for diagnosis. J. Dermatol., 38: 715, 2011.

30- LENCASTRE A. and TOSTI A.: Role of trichoscopy in children's scalp and hair disorders. Pediatr. Dermatol., 30 (6): 674-82, 2013.

31- LACARRUBBA F., D'AMICO V., NASCA M.R., et al.: Use of dermatoscopy and videodermatoscopy in therapeutic follow-up: A review. Int. J. Dermatol., 49: 866-73, 2010.

32- EL-TAWEEL A., EL-ESAWY F. and ABDEL-SALAM O.: Different trichoscopic features of tinea capitis and alopecia areata in pediatric patients. Dermatol. Research Practice, P. 1-6, 2014. 


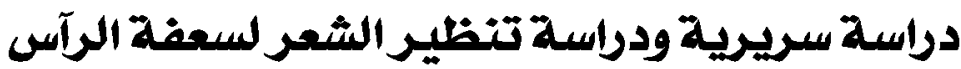 مقارنة بدآيء الثعلبة فئى الآطفال}

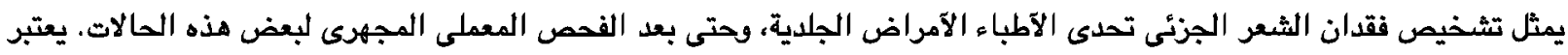

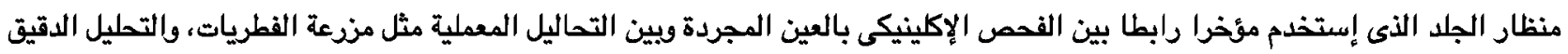

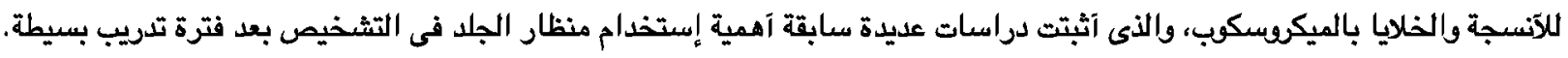

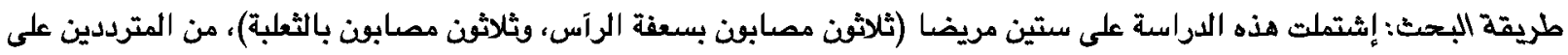

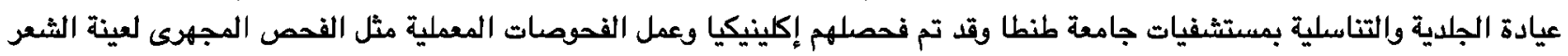
بإستخدام . 1\% هيدركسيد البوتاسيوم وكذلك مزرعة الفطريات لتاكيد التشخيص الإكلينيكى. وآيضا تم إستخدام منظار الجلد وذلك اللوصول إلى السمات الديرموسكوبية المختلة لمرض سعفة الرآس ومرض الثعلبة.

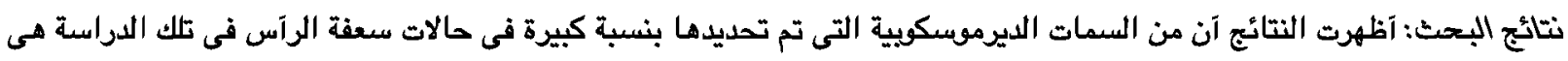

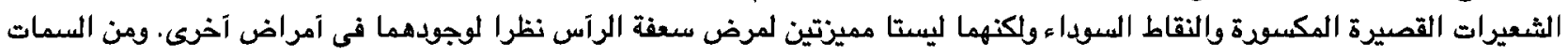

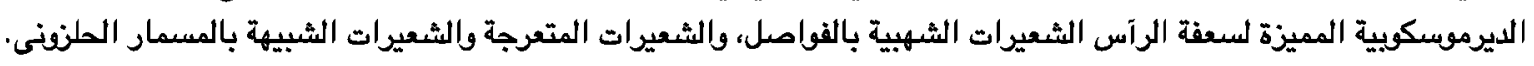

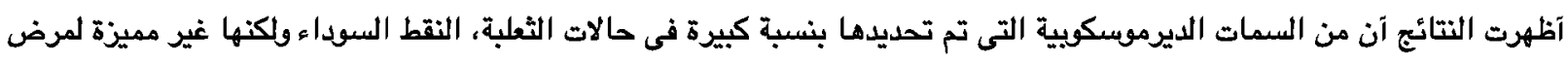

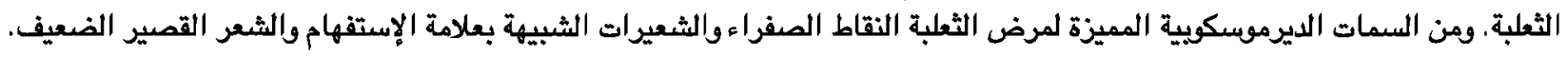
وآستتج من هذه الدراسة: آهمية إستخدام منظار الجلد فى تشخيص آمراض فقدان الشعر الجزئى، وإمكانية إستخدام المنظار فى التقريق بين سعفة الرآس والثعلبة. 